\title{
The existence and exponential stability of random impulsive fractional differential equations
}

\author{
Suping Zhang ${ }^{1 *}$ and Wei Jiang ${ }^{2}$
}

"Correspondence: zsp606@163.com 'School of Mathematics and Physics, Anhui Jianzhu University, Hefei, P.R. China

Full list of author information is available at the end of the article

\begin{abstract}
In this paper, the existence and exponential stability of mild solutions of random impulsive fractional differential equations are studied. The results are obtained by using the Leray-Schauder alternative fixed point theorem.
\end{abstract}

Keywords: Random impulse; Fractional differential equations; Exponential stability; Leray-Schauder alternative fixed point

\section{Introduction}

For impulsive differential systems, most published papers deal with the problem with fixed time impulses [1-3]. However, actual jumps do not always happen at fixed points but usually at random points. The solutions of random impulsive differential equations is a stochastic process. It is different from deterministic impulsive differential equations and also it is different from stochastic differential equations. At present, the properties of solutions to some integer order differential equations with random impulses have been studied [4-8]. The existence, uniqueness, and stability of fractional differential equations without random impulses have been shown by many authors [9-18]. However, the properties of solutions to fractional delay differential equations with random impulses have not been studied. In [19], the authors study the existence, uniqueness, and stability through continuous dependence on initial conditions and Hyers-Ulam-Rassias stability for random impulsive fractional differential equations, but in that paper, the authors did not consider delay. In some applications, besides impulsive effects, delay effects cannot be ignored.

Motivated by the above consideration, we consider fractional differential equations with random impulses of the form

$$
\begin{aligned}
& { }^{c} D_{t}^{q} x(t)=A x(t)+f\left(t, x_{t}\right), \quad t \neq \xi_{k}, t \geq t_{0}, \\
& x\left(\xi_{k}\right)=b_{k}\left(\tau_{k}\right) x\left(\xi_{k}^{-}\right), \quad k=1,2, \ldots, \\
& x_{t_{0}}=\varphi .
\end{aligned}
$$

Let $X$ be a real separable Hilbert space and $\Omega$ be a nonempty set. For the sake of simplicity, we denote $R_{\tau}=[\tau,+\infty), R^{+}=[0,+\infty)$. Assume that $\tau_{k}$ is a random variable defined from $\Omega$ to $D_{k} \stackrel{\text { def. }}{=}\left(0, d_{k}\right)$ for $k=1,2, \ldots$, where $0<d_{k}<+\infty$. Furthermore, assume that $\tau_{i}$

(c) The Author(s) 2018. This article is distributed under the terms of the Creative Commons Attribution 4.0 International License (http://creativecommons.org/licenses/by/4.0/), which permits unrestricted use, distribution, and reproduction in any medium, provided you give appropriate credit to the original author(s) and the source, provide a link to the Creative Commons license, and indicate if changes were made. 
and $\tau_{j}$ are independent of each other as $i \neq j$ for $i, j=1,2, \ldots \mathrm{A}$ is the infinitesimal generator of a strongly continuous semigroup of bounded linear operators $S(t)$ with domain $D(A) \subset X$; the functional $f: R^{+} \times C \rightarrow X, C=C([-r, 0], X)$ is the set of piecewise continuous functions, $r>0 ; x_{t}$ is a function when $\mathrm{t}$ is fixed, $x_{t}(\theta)=x(t+\theta), \theta \in[-r, 0] ; \xi_{0}=t_{0}$ and $\xi_{k}=\xi_{k-1}+\tau_{k}$ for $k=1,2, \ldots$, here $t_{0} \in R_{\tau}$ is an arbitrary given real number. The impulse moments $\xi_{k}$ form a strictly increasing sequence, i.e., $t_{0}=\xi_{0}<\xi_{1}<\xi_{2}<\cdots<\lim _{k \rightarrow \infty} \xi_{k}$; $b_{k}: D_{k} \rightarrow X$ for each $k=1,2, \ldots ; x\left(\xi_{k}^{-}\right)=\lim _{t \rightarrow \xi_{k}} x(t)$ according to their paths with the norm $\|x\|_{t}=\sup _{t-r \leq s \leq t}|x(s)|$ for each $\mathrm{t}$ satisfying $t \geq t_{0} ;\|\cdot\|$ is any given norm in $\mathrm{X}$; $\varphi$ is a function defined from $[-r, 0]$ to $\mathrm{X} ;{ }^{c} D^{q}$ is the Caputo fractional derivative of order $0<q<1$.

This paper studies the properties of solutions to system (1.1) by using the theory of functional differential equation, fractional differential equation, and stochastic analysis techniques. Firstly, we investigate the existence of a mild solution for (1.1) by using the LeraySchauder alternative fixed point theorem. Secondly, we study the exponential stability in the quadratic mean of (1.1). Finally, an example is presented to illustrate our results.

\section{Some preliminaries}

In this section, we shall recall some basic definitions and lemma which will be used in this paper.

Denote by $\left\{B_{t}, t \geq 0\right\}$ the simple counting process generated by $\left\{\xi_{n}\right\}$, that is, $\left\{B_{t} \geq n\right\}=$ $\left\{\xi_{n} \leq t\right\}$, and denote by $F_{t}$ the $\sigma$-algebra generated by $\left\{B_{t}, t \geq 0\right\}$. Then $\left(\Omega, P,\left\{F_{t}\right\}\right)$ is a probability space. Let $L_{2}=L_{2}\left(\Omega, F_{t}, X\right)$ denote the Hilbert space of all $F_{t}$-measurable square integrable random variables with values in $\mathrm{X}$.

Assume that $T>t_{0}$ is any fixed time to be determined later and $B$ denotes the Banach space $\beta\left(\left[t_{0}-r, T\right], L_{2}\right)$, the family of all $F_{t}$ - measurable, $C$-valued random variables $\varphi$ with the norm

$$
\|\varphi\|_{B}^{2}=\sup _{t_{0} \leq t \leq T} E\|\varphi\|_{t}^{2}
$$

Let $L_{2}^{0}(\Omega, \beta)$ denote the family of all $F_{0}$-measurable, $\beta$-valued random variables $\varphi$.

Definition 2.1 The fractional integral of order q with the lower limit 0 for a function $f$ is defined as

$$
I^{q} f(t)=\frac{1}{\Gamma(q)} \int_{0}^{t} \frac{f(s)}{(t-s)^{1-q}} d s, \quad t>0, q>0
$$

provided the right-hand side is pointwise defined on $[0, \infty)$, where $\Gamma$ is the gamma function.

Definition 2.2 The Riemann-Liouville derivative of order q with the lower limit 0 for a function $f:[0, \infty) \rightarrow R$ can be written as

$$
{ }^{L} D^{q} f(t)=\frac{1}{\Gamma(n-q)} \frac{d^{n}}{d t^{n}} \int_{0}^{t} \frac{f(s)}{(t-s)^{q+1-n}} d s, \quad t>0, n-1<q<n .
$$


Definition 2.3 The Caputo derivative of order q for a function $f:[0, \infty) \rightarrow R$ can be written as

$$
{ }^{c} D^{q} f(t)={ }^{L} D^{q}\left[f(t)-\sum_{k=0}^{n-1} \frac{t^{k}}{k !} f^{(k)}(0)\right], \quad t>0, n-1<q<n .
$$

Definition 2.4 A semigroup $\left\{S(t), t \geq t_{0}\right\}$ is said to be uniformly bounded if $\|S(t)\| \leq M$ for all $t \geq t_{0}$, where $M \geq 1$ is some constant. If $M=1$, then the semigroup is said to be contraction semigroup.

Definition 2.5 A map $f:[\tau, T] \times C \rightarrow X$ is said to be $L^{2}$-Carathéodory if

(i) $t \rightarrow f(t, u)$ is measurable for each $u \in C$;

(ii) $u \rightarrow f(t, u)$ is continuous for almost all $t \in[\tau, T]$;

(iii) for each positive integer $m>0$, there exists $\alpha_{m} \in L^{1}\left([\tau, T], R^{+}\right)$such that

$$
\sup _{\|u\|_{B} \leq m}\|f(t, u)\|^{2} \leq \alpha_{m}(t) \quad \text { for } t \in[\tau, T] \text {, a.e. }
$$

Definition 2.6 For a given $T \in\left(t_{0},+\infty\right)$, a stochastic process $\left\{x(t) \in \beta, t_{0}-r \leq t \leq T\right\}$ is called a mild solution to Eq. (1.1) in $\left(\Omega, P,\left\{F_{t}\right\}\right)$ if

(i) $x(t) \in \beta$ is $F_{t}$-adapted for $t \geq t_{0}$;

(ii) $x\left(t_{0}+s\right)=\varphi(s) \in L_{2}^{0}(\Omega, \beta)$, when $s \in[-r, 0]$, and

$$
\begin{aligned}
x(t)= & \sum_{k=0}^{+\infty}\left[\prod_{i=1}^{k} b_{i}\left(\tau_{i}\right) S\left(t-t_{0}\right) \varphi(0)\right. \\
& +\frac{1}{\Gamma(q)} \sum_{i=1}^{k} \prod_{j=i}^{k} b_{j}\left(\tau_{j}\right) \int_{\xi_{i-1}}^{\xi_{i}}(t-s)^{q-1} S(t-s) f\left(s, x_{s}\right) d s \\
& \left.+\frac{1}{\Gamma(q)} \int_{\xi_{k}}^{t}(t-s)^{q-1} S(t-s) f\left(s, x_{s}\right) d s\right] I_{\left[\xi_{k}, \xi_{k+1}\right)}(t), \quad t \in\left[t_{0}, T\right],
\end{aligned}
$$

where $\prod_{j=m}^{n}(\cdot)=1$ as $m>n, \prod_{j=i}^{k} b_{j}\left(\tau_{j}\right)=b_{k}\left(\tau_{k}\right) b_{k-1}\left(\tau_{k-1}\right) \cdots b_{i}\left(\tau_{i}\right)$, and $I_{A}(\cdot)$ is the index function, i.e.,

$$
I_{A}(t)= \begin{cases}1, & \text { if } t \in A \\ 0, & \text { if } t \notin A .\end{cases}
$$

Our existence and exponential stability theorems are based on the following theorem, which is a version of the topological transversality theorem.

Lemma 2.1 Let $E$ be a convex subset of a Banach space $X$, and assume that $0 \in E$. Let $F: E \rightarrow E$ be a completely continuous operator, and let

$$
U(F)=\{x \in E: x=\lambda F x \text { for some } 0<\lambda<1\},
$$

then either $U(F)$ is unbounded or $F$ has a fixed point. 


\section{Existence results}

In this section, we prove the existence of a mild solution of system (1.1) by using the following hypotheses:

$\left(H_{1}\right)$ There exist a continuous non-decreasing function $H: R^{+} \rightarrow(0, \infty)$ and $\delta \in$ $L^{1}\left([\tau, T], R^{+}\right)$such that

$$
E\left\|f\left(t, \psi_{t}\right)\right\|^{2} \leq \delta(t) H\left(E\|\psi\|_{t}^{2}\right)
$$

for every $t \in[\tau, T]$ and for any stochastic process $\psi \in C$.

$\left(H_{2}\right) \max _{i, k}\left\{\prod_{j=i}^{k}\left\|b_{j}\left(\tau_{j}\right)\right\|\right\}$ is uniformly bounded, that is, there is $B>0$ such that

$$
\max _{i, k}\left\{\prod_{j=i}^{k}\left\|b_{j}\left(\tau_{j}\right)\right\|\right\} \leq B
$$

for all $\tau_{j} \in D_{j}, j=1,2, \ldots$.

Theorem 3.1 Assume $\left(H_{1}\right)-\left(H_{2}\right)$ hold, then system (1.1) has a mild solution $x(t)$, defined on $\left[t_{0}, T\right]$, provided that the following inequality is satisfied:

$$
M_{1} \int_{t_{0}}^{T} \delta(s) d s<\int_{c_{1}}^{\infty} \frac{d s}{H(s)},
$$

where $M_{1}=2 M^{2} \max \left\{1, B^{2}\right\} \frac{\left(T-t_{0}\right)^{2 q-1}}{(2 q-1) \Gamma(q)}, c_{1}=2 M^{2} B^{2} E\|\varphi\|^{2}$, and $M C \geq \frac{1}{\sqrt{2}}$.

Proof Let $T$ be an arbitrary positive number $t_{0}<T<+\infty$. Define an operator $\Phi: \beta \rightarrow \beta$ as follows:

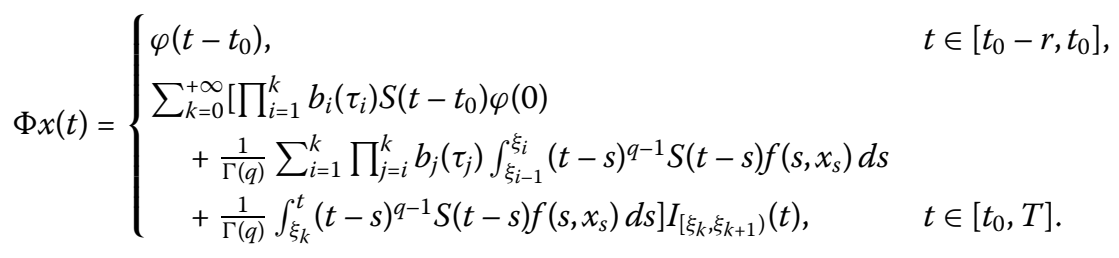

First we establish the a priori estimates for the solutions of the integral equation and $\lambda \in(0,1):$

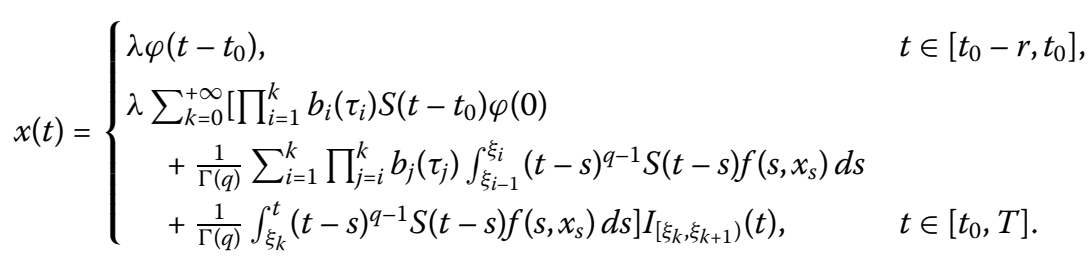

Thus by $\left(H_{1}\right)-\left(H_{2}\right)$, we have

$$
\begin{aligned}
& \|x(t)\|^{2} \\
& \leq \lambda^{2}\left[\sum _ { k = 0 } ^ { + \infty } \left[\left\|\prod_{i=1}^{k} b_{i}\left(\tau_{i}\right)\right\|\left\|S\left(t-t_{0}\right)\right\|\|\varphi(0)\|\right.\right.
\end{aligned}
$$




$$
\begin{array}{rl} 
& +\frac{1}{\Gamma(q)} \sum_{i=1}^{k}\left\|\prod_{j=i}^{k} b_{j}\left(\tau_{j}\right)\right\| \int_{\xi_{i-1}}^{\xi_{i}}(t-s)^{q-1}\left\|S(t-s) f\left(s, x_{s}\right)\right\| d s \\
& \left.\left.+\frac{1}{\Gamma(q)} \int_{\xi_{k}}^{t}(t-s)^{q-1}\left\|S(t-s) f\left(s, x_{s}\right)\right\| d s\right] I_{\left[\xi_{k}, \xi_{k+1}\right.}(t)\right]^{2} \\
\leq & 2 \sum_{k=0}^{+\infty}\left[\left\|\prod_{i=1}^{k} b_{i}\left(\tau_{i}\right)\right\|^{2}\left\|S\left(t-t_{0}\right)\right\|^{2}\|\varphi(0)\|^{2} I_{\left[\xi_{k}, \xi_{k+1}\right)}(t)\right] \\
& +2\left[\sum _ { k = 0 } ^ { \infty } \left[\frac{1}{\Gamma(q)} \sum_{i=1}^{k}\left\|\prod_{j=i}^{k} b_{j}\left(\tau_{j}\right)\right\| \int_{\xi_{i-1}}^{\xi_{i}}(t-s)^{q-1}\left\|S(t-s) f\left(s, x_{s}\right)\right\| d s\right.\right. \\
& \left.\left.\left.+\frac{1}{\Gamma(q)} \int_{\xi_{k}}^{t}(t-s)^{q-1}\left\|S(t-s) f\left(s, x_{s}\right)\right\| d s\right] I_{\left[\xi_{k}, \xi_{k+1}\right.}\right)\right]^{2} \\
\leq & 2 M^{2} B^{2}\|\varphi(0)\|^{2}+2 M^{2} \max \left\{1, B^{2}\right\} \frac{\left(T-t_{0}\right)^{2 q-1}}{\Gamma(q)(2 q-1)} \int_{t_{0}}^{t}\left\|f\left(s, x_{s}\right)\right\|^{2} d s, \\
\|x\|_{t}^{2} \leq & 2 M^{2} B^{2}\|\varphi\|^{2}+2 M^{2} \max \left\{1, B^{2}\right\} \frac{\left(T-t_{0}\right)^{2 q-1}}{\Gamma(q)(2 q-1)} \int_{t_{0}}^{t}\left\|f\left(s, x_{s}\right)\right\|^{2} d s, \\
E\|x\|_{t}^{2} & \\
\leq & 2 M^{2} B^{2} E\left[\|\varphi\|^{2}\right]+2 M^{2} \max \left\{1, B^{2}\right\} \frac{\left(T-t_{0}\right)^{2 q-1}}{\Gamma(q)(2 q-1)} \int_{t_{0}}^{t} E\left[\left\|f\left(s, x_{s}\right)\right\|^{2}\right] d s \\
\leq & 2 M^{2} B^{2} E\left[\|\varphi\|^{2}\right]+2 M^{2} \max \left\{1, B^{2}\right\} \frac{\left(T-t_{0}\right)^{2 q-1}}{\Gamma(q)(2 q-1)} \int_{t_{0}}^{t} \delta(s) H\left(E\left[\|x\|_{s}^{2}\right]\right) d s, \\
\sup _{0} \leq v \leq t & E\|x\|_{v}^{2} \\
\leq & 2 M^{2} B^{2} E\left[\|\varphi\|^{2}\right] \\
+ & 2 M^{2} \max \left\{1, B^{2}\right\} \frac{\left(T-t_{0}\right)^{2 q-1}}{\Gamma(q)(2 q-1)} \int_{t_{0}}^{t} \delta(s) H\left(\sup _{t_{0} \leq v \leq s} E\left[\|x\|_{v}^{2}\right]\right) d s . \\
&
\end{array}
$$

Let

$$
\ell(t)=\sup _{t_{0} \leq v \leq t} E\left[\|x\|_{v}^{2}\right], \quad t \in\left[t_{0}, T\right]
$$

Then, for any $t \in\left[t_{0}, T\right]$, it follows that

$$
\ell(t) \leq 2 M^{2} B^{2} E\left[\|\varphi\|^{2}\right]+2 M^{2} \max \left\{1, B^{2}\right\} \frac{\left(T-t_{0}\right)^{2 q-1}}{\Gamma(q)(2 q-1)} \int_{t_{0}}^{t} \delta(s) H(\ell(s)) d s .
$$

Denoting by $u(t)$ the right-hand side of the above inequality, we obtain that

$$
\begin{aligned}
& \ell(t) \leq u(t), \quad t \in\left[t_{0}, T\right], \\
& u\left(t_{0}\right)=2 M^{2} B^{2} E\|\varphi\|^{2}=c_{1}
\end{aligned}
$$


and

$$
\begin{aligned}
u^{\prime}(t) & =2 M^{2} \max \left\{1, B^{2}\right\} \frac{\left(T-t_{0}\right)^{2 q-1}}{\Gamma(q)(2 q-1)} \delta(t) H(\ell(t)) \\
& \leq 2 M^{2} \max \left\{1, B^{2}\right\} \frac{\left(T-t_{0}\right)^{2 q-1}}{\Gamma(q)(2 q-1)} \delta(t) H(u(t)), \quad t \in\left[t_{0}, T\right] .
\end{aligned}
$$

Then

$$
\frac{u^{\prime}(t)}{H(u(t))} \leq 2 M^{2} \max \left\{1, B^{2}\right\} \frac{\left(T-t_{0}\right)^{2 q-1}}{\Gamma(q)(2 q-1)} \delta(t), \quad t \in\left[t_{0}, T\right]
$$

Integrating the above inequality from $t_{0}$ to $t$ and making use of the change of variable, we obtain

$$
\begin{aligned}
\int_{u\left(t_{0}\right)}^{u(t)} \frac{d s}{H(s)} & \leq 2 M^{2} \max \left\{1, B^{2}\right\} \frac{\left(T-t_{0}\right)^{2 q-1}}{\Gamma(q)(2 q-1)} \int_{t_{0}}^{t} \delta(s) d s \\
& \leq 2 M^{2} \max \left\{1, B^{2}\right\} \frac{\left(T-t_{0}\right)^{2 q-1}}{\Gamma(q)(2 q-1)} \int_{t_{0}}^{T} \delta(s) d s \\
& <\int_{c_{1}}^{\infty} \frac{d s}{H(s)}=\int_{u\left(t_{0}\right)}^{\infty} \frac{d s}{H(s)} .
\end{aligned}
$$

From the above inequality and by the mean value theorem, there is a constant $K$ such that $u(t) \leq K$, and hence $\ell(t) \leq K$. Since $\sup _{t_{0} \leq v \leq t} E\left[\|x\|_{v}^{2}\right]=\ell(t)$ holds for every $t \in\left[t_{0}, T\right]$, we have $\sup _{t_{0} \leq v \leq T} E\left[\|x\|_{v}^{2}\right] \leq K$, where $K$ only depends on $T$ and the functions $\delta$ and $H$, consequently

$$
E\|x\|_{B}^{2}=\sup _{t_{0} \leq v \leq T} E\|x\|_{v}^{2} \leq K
$$

In the next steps, we will prove that $\Phi$ is continuous and completely continuous.

Step 1 . We prove that $\Phi$ is continuous.

Let $\left\{x_{n}\right\}$ be a convergent sequence of elements of $x$ in $\beta$. Then, for each $t \in\left[t_{0}, T\right]$, we have

$$
\begin{aligned}
\Phi x_{n}(t)= & \sum_{k=0}^{+\infty}\left[\prod_{i=1}^{k} b_{i}\left(\tau_{i}\right) S\left(t-t_{0}\right) \varphi(0)\right. \\
& +\frac{1}{\Gamma(q)} \sum_{i=1}^{k} \prod_{j=i}^{k} b_{j}\left(\tau_{j}\right) \int_{\xi_{i-1}}^{\xi_{i}}(t-s)^{q-1} S(t-s) f\left(s, x_{n_{s}}\right) d s \\
& \left.+\frac{1}{\Gamma(q)} \int_{\xi_{k}}^{t}(t-s)^{q-1} S(t-s) f\left(s, x_{n_{s}}\right) d s\right] I_{\left[\xi_{k}, \xi_{k+1}\right)}(t) .
\end{aligned}
$$

Thus

$$
\Phi x_{n}(t)-\Phi x(t)=\sum_{k=0}^{+\infty}\left[\frac{1}{\Gamma(q)} \sum_{i=1}^{k} \prod_{j=i}^{k} b_{j}\left(\tau_{j}\right) \int_{\xi_{i-1}}^{\xi_{i}}(t-s)^{q-1} S(t-s)\left\{f\left(s, x_{n_{s}}\right)-f\left(s, x_{s}\right)\right\} d s\right.
$$




$$
\left.+\frac{1}{\Gamma(q)} \int_{\xi_{k}}^{t}(t-s)^{q-1} S(t-s)\left\{f\left(s, x_{n_{s}}\right)-f\left(s, x_{s}\right)\right\} d s\right] I_{\left[\xi_{k}, \xi_{k+1}\right)}(t)
$$

and

$$
E\left\|\Phi x_{n}-\Phi x\right\|_{t}^{2} \leq M^{2} \max \left\{1, B^{2}\right\} \frac{\left(T-t_{0}\right)^{2 q-1}}{\Gamma(q)(2 q-1)} \int_{t_{0}}^{t} E\left\|f\left(s, x_{n_{s}}\right)-f\left(s, x_{s}\right)\right\|^{2} d s
$$

Thus $\Phi$ is continuous.

Step 2 . We prove that $\Phi$ is a completely continuous operator.

Denote

$$
B_{m}=\left\{x \in \beta \mid\|x\|_{\beta}^{2} \leq m\right\}
$$

for some $m \geq 0$.

Step 2.1. We show that $\Phi$ maps $B_{m}$ into an equicontinuous family.

Let $x \in B_{m}$ and $t_{1}, t_{2} \in\left[t_{0}, T\right]$. If $t_{0}<t_{1}<t_{2}<T$, then by using $\left(H_{1}\right)-\left(H_{2}\right)$ and condition

(3.1), we have

$$
\begin{aligned}
\Phi x\left(t_{2}\right) & -\Phi x\left(t_{1}\right) \\
= & \sum_{k=0}^{+\infty}\left[\prod_{i=1}^{k} b_{i}\left(\tau_{i}\right) S\left(t_{2}-t_{0}\right) \varphi(0)\right. \\
& +\frac{1}{\Gamma(q)} \sum_{i=1}^{k} \prod_{j=i}^{k} b_{j}\left(\tau_{j}\right) \int_{\xi_{i-1}}^{\xi_{i}}\left(t_{2}-s\right)^{q-1} S\left(t_{2}-s\right) f\left(s, x_{s}\right) d s \\
& \left.+\frac{1}{\Gamma(q)} \int_{\xi_{k}}^{t_{2}}\left(t_{2}-s\right)^{q-1} S\left(t_{2}-s\right) f\left(s, x_{s}\right) d s\right] I_{\left[\xi_{k}, \xi_{k+1}\right)}\left(t_{2}\right) \\
& -\sum_{k=0}^{+\infty}\left[\prod_{i=1}^{k} b_{i}\left(\tau_{i}\right) S\left(t_{1}-t_{0}\right) \varphi(0)\right. \\
& +\frac{1}{\Gamma(q)} \sum_{i=1}^{k} \prod_{j=i}^{k} b_{j}\left(\tau_{j}\right) \int_{\xi_{i-1}}^{\xi_{i}}\left(t_{1}-s\right)^{q-1} S\left(t_{1}-s\right) f\left(s, x_{s}\right) d s \\
& \left.+\frac{1}{\Gamma(q)} \int_{\xi_{k}}^{t_{2}}\left(t_{2}-s\right)^{q-1} S\left(t_{2}-s\right) f\left(s, x_{s}\right) d s\right]\left(I_{\left[\xi_{k}, \xi_{k+1}\right)}\left(t_{2}\right)-I_{\left[\xi_{k}, \xi_{k+1}\right)}\left(t_{1}\right)\right) \\
& \left.+\frac{1}{\Gamma(q)} \int_{\xi_{k}}^{t_{1}}\left(t_{1}-s\right)^{q-1} S\left(t_{1}-s\right) f\left(s, x_{s}\right) d s\right] I_{\left[\xi_{k}, \xi_{k+1}\right)}\left(t_{1}\right) \\
= & \sum_{k=0}^{+\infty}\left[\prod_{i=1}^{k} b_{i}\left(\tau_{i}\right) S\left(t_{2}-t_{0}\right) \varphi\left(S\left(t_{2}-t_{0}\right)-S\left(t_{1}-t_{0}\right)\right] \varphi(0)\right. \\
& \frac{1}{\Gamma(q)} \sum_{i=1}^{k} \prod_{j=i}^{k} b_{j}\left(\tau_{j}\right) \int_{\xi_{i-1}}^{\xi_{i}}\left(t_{2}-s\right)^{q-1} S\left(t_{2}-s\right) f\left(s, x_{s}\right) d s
\end{aligned}
$$




$$
\begin{aligned}
& +\frac{1}{\Gamma(q)} \sum_{i=1}^{k} \prod_{j=i}^{k} b_{j}\left(\tau_{j}\right) \int_{\xi_{i-1}}^{\xi_{i}}\left[\left(t_{2}-s\right)^{q-1} S\left(t_{2}-s\right)-\left(t_{1}-s\right)^{q-1} S\left(t_{1}-s\right)\right] f\left(s, x_{s}\right) d s \\
& +\frac{1}{\Gamma(q)} \int_{\xi_{k}}^{t_{1}}\left[\left(t_{2}-s\right)^{q-1} S\left(t_{2}-s\right)-\left(t_{1}-s\right)^{q-1} S\left(t_{1}-s\right)\right] f\left(s, x_{s}\right) d s \\
& \left.+\frac{1}{\Gamma(q)} \int_{t_{1}}^{t_{2}}\left(t_{2}-s\right)^{q-1} S\left(t_{2}-s\right) f\left(s, x_{s}\right) d s\right] I_{\left[\xi_{k}, \xi_{k+1}\right)}\left(t_{1}\right) .
\end{aligned}
$$

Then

$$
E\left\|\Phi x\left(t_{2}\right)-\Phi x\left(t_{1}\right)\right\|^{2} \leq 2 E\left\|I_{1}\right\|^{2}+2 E\left\|I_{2}\right\|^{2},
$$

where

$$
\begin{aligned}
I_{1}= & \sum_{k=0}^{+\infty}\left[\prod_{i=1}^{k} b_{i}\left(\tau_{i}\right) S\left(t_{2}-t_{0}\right) \varphi(0)\right. \\
& +\frac{1}{\Gamma(q)} \sum_{i=1}^{k} \prod_{j=i}^{k} b_{j}\left(\tau_{j}\right) \int_{\xi_{i-1}}^{\xi_{i}}\left(t_{2}-s\right)^{q-1} S\left(t_{2}-s\right) f\left(s, x_{s}\right) d s \\
& \left.+\frac{1}{\Gamma(q)} \int_{\xi_{k}}^{t_{2}}\left(t_{2}-s\right)^{q-1} S\left(t_{2}-s\right) f\left(s, x_{s}\right) d s\right]\left(I_{\left[\xi_{k}, \xi_{k+1}\right)}\left(t_{2}\right)-I_{\left[\xi_{k}, \xi_{k+1}\right)}\left(t_{1}\right)\right)
\end{aligned}
$$

and

$$
\begin{aligned}
I_{2}= & \sum_{k=0}^{+\infty}\left[\prod_{i=1}^{k} b_{i}\left(\tau_{i}\right)\left[S\left(t_{2}-t_{0}\right)-S\left(t_{1}-t_{0}\right)\right] \varphi(0)\right. \\
& +\frac{1}{\Gamma(q)} \sum_{i=1}^{k} \prod_{j=i}^{k} b_{j}\left(\tau_{j}\right) \int_{\xi_{i-1}}^{\xi_{i}}\left[\left(t_{2}-s\right)^{q-1} S\left(t_{2}-s\right)-\left(t_{1}-s\right)^{q-1} S\left(t_{1}-s\right)\right] f\left(s, x_{s}\right) d s \\
& +\frac{1}{\Gamma(q)} \int_{\xi_{k}}^{t_{1}}\left[\left(t_{2}-s\right)^{q-1} S\left(t_{2}-s\right)-\left(t_{1}-s\right)^{q-1} S\left(t_{1}-s\right)\right] f\left(s, x_{s}\right) d s \\
& \left.+\frac{1}{\Gamma(q)} \int_{t_{1}}^{t_{2}}\left(t_{2}-s\right)^{q-1} S\left(t_{2}-s\right) f\left(s, x_{s}\right) d s\right] I_{\left[\xi_{k}, \xi_{k+1}\right)}\left(t_{1}\right) .
\end{aligned}
$$

Furthermore,

$$
\begin{aligned}
E\left\|I_{1}\right\|^{2} \leq & E\left(\sum _ { k = 0 } ^ { + \infty } \left[\prod_{i=1}^{k}\left\|b_{i}\left(\tau_{i}\right)\right\|\left\|S\left(t_{2}-t_{0}\right)\right\|\|\varphi(0)\|\right.\right. \\
& +\frac{1}{\Gamma(q)} \sum_{i=1}^{k}\left\|\prod_{j=i}^{k} b_{j}\left(\tau_{j}\right)\right\| \int_{\xi_{i-1}}^{\xi_{i}}\left(t_{2}-s\right)^{q-1}\left\|S\left(t_{2}-s\right)\right\|\left\|f\left(s, x_{s}\right)\right\| d s \\
& \left.\left.+\frac{1}{\Gamma(q)} \int_{\xi_{k}}^{t_{2}}\left(t_{2}-s\right)^{q-1}\left\|S\left(t_{2}-s\right)\right\|\left\|f\left(s, x_{s}\right)\right\| d s\right]\left(I_{\left[\xi_{k}, \xi_{k+1}\right)}\left(t_{2}\right)-I_{\left[\xi_{k}, \xi_{k+1}\right)}\left(t_{1}\right)\right)\right)^{2} \\
\leq & 2 M^{2} B^{2} E\left\|_{\varphi(0)}\right\|^{2} E\left(I_{\left[\xi_{k}, \xi_{k+1}\right)}\left(t_{2}\right)-I_{\left[\xi_{k}, \xi_{k+1}\right)}\left(t_{1}\right)\right)
\end{aligned}
$$




$$
\begin{aligned}
& +2 \max \left\{1, B^{2}\right\} \frac{\left(T-t_{0}\right)^{2 q-1}}{\Gamma(q)(2 q-1)} E \int_{t_{0}}^{t_{2}}\left\|S\left(t_{2}-s\right)\right\|\left\|f\left(s, x_{s}\right)\right\| d s E\left(I_{\left[\xi_{k}, \xi_{k+1}\right)}\left(t_{2}\right)\right. \\
& \left.-I_{\left[\xi_{k}, \xi_{k+1}\right)}\left(t_{1}\right)\right) \\
\leq & 2 M^{2} B^{2} E\|\varphi(0)\|^{2} E\left(I_{\left[\xi_{k}, \xi_{k+1}\right)}\left(t_{2}\right)-I_{\left[\xi_{k}, \xi_{k+1}\right)}\left(t_{1}\right)\right) \\
& +2 M^{2} \max \left\{1, B^{2}\right\} \frac{\left(T-t_{0}\right)^{2 q-1}}{\Gamma(q)(2 q-1)} \int_{t_{0}}^{t_{2}} \delta(s) H\left(E\|x\|_{s}^{2}\right) d s E\left(I_{\left[\xi_{k}, \xi_{k+1}\right)}\left(t_{2}\right)\right. \\
& \left.-I_{\left[\xi_{k}, \xi_{k+1}\right)}\left(t_{1}\right)\right) \\
\leq & 2 M^{2} B^{2} E\|\varphi(0)\|^{2} E\left(I_{\left[\xi_{k}, \xi_{k+1}\right)}\left(t_{2}\right)-I_{\left[\xi_{k}, \xi_{k+1}\right)}\left(t_{1}\right)\right) \\
& +2 M^{2} \max \left\{1, B^{2}\right\} \frac{\left(T-t_{0}\right)^{2 q-1}}{\Gamma(q)(2 q-1)} \int_{t_{0}}^{t_{2}} M^{*} H(E(m)) d s E\left(I_{\left[\xi_{k}, \xi_{k+1}\right)}\left(t_{2}\right)\right. \\
& \left.-I_{\left[\xi_{k}, \xi_{k+1}\right)}\left(t_{1}\right)\right) \\
\rightarrow & 0, \quad \text { as } t_{1} \rightarrow t_{2}
\end{aligned}
$$

where $M^{*}=\sup \left\{\delta(t): t \in\left[t_{0}, T\right]\right\}$.

$$
\begin{aligned}
E\left\|I_{2}\right\|^{2} \leq & E\left(\sum _ { k = 0 } ^ { + \infty } \left[\prod_{i=1}^{k}\left\|b_{i}\left(\tau_{i}\right)\right\|\left\|S\left(t_{2}-t_{0}\right)-S\left(t_{1}-t_{0}\right)\right\|\|\varphi(0)\|\right.\right. \\
& +\frac{1}{\Gamma(q)} \sum_{i=1}^{k} \prod_{j=i}^{k}\left\|b_{j}\left(\tau_{j}\right)\right\| \int_{\xi_{i-1}}^{\xi_{i}} \|\left(t_{2}-s\right)^{q-1} S\left(t_{2}-s\right) \\
& -\left(t_{1}-s\right)^{q-1} S\left(t_{1}-s\right)\|\| f\left(s, x_{s}\right) \| d s \\
& +\frac{1}{\Gamma(q)} \int_{\xi_{k}}^{t_{1}}\left\|\left(t_{2}-s\right)^{q-1} S\left(t_{2}-s\right)-\left(t_{1}-s\right)^{q-1} S\left(t_{1}-s\right)\right\|\left\|f\left(s, x_{s}\right)\right\| d s \\
& \left.\left.+\frac{1}{\Gamma(q)} \int_{t_{1}}^{t_{2}}\left(t_{2}-s\right)^{q-1}\left\|S\left(t_{2}-s\right)\right\|\left\|f\left(s, x_{s}\right)\right\| d s\right] I_{\left[\xi_{k}, \xi_{k+1}\right)}\left(t_{1}\right)\right)^{2} \\
\leq & 3 B^{2}\left\|S\left(t_{2}-t_{0}\right)-S\left(t_{1}-t_{0}\right)\right\|^{2} E\|\varphi(0)\|^{2} \\
& +3 \max \left\{1, B^{2}\right\}\left(t_{1}-t_{0}\right) \frac{1}{\Gamma(q)} E \int_{t_{0}}^{t_{1}} \|\left(t_{2}-s\right)^{q-1} S\left(t_{2}-s\right) \\
& -\left(t_{1}-s\right)^{q-1} S\left(t_{1}-s\right)\left\|^{2}\right\| f\left(s, x_{s}\right) \|^{2} d s \\
& +3 M^{2} \frac{\left(T-t_{0}\right)^{2 q-1}}{\Gamma(q)(2 q-1)} E \int_{t_{1}}^{t_{2}}\left\|f\left(s, x_{s}\right)\right\|^{2} d s \\
\leq & 3 B^{2}\left\|S\left(t_{2}-t_{0}\right)-S\left(t_{1}-t_{0}\right)\right\|^{2} E\|\varphi(0)\|^{2} \\
& +3 \max \left\{1, B^{2}\right\}\left(t_{1}-t_{0}\right) \frac{1}{\Gamma(q)} \int_{t_{0}}^{t_{1}} \|\left(t_{2}-s\right)^{q-1} S\left(t_{2}-s\right) \\
& -\left(t_{1}-s\right)^{q-1} S\left(t_{1}-s\right) \|^{2} M^{*} H(m) d s \\
+ & 3 M^{2} \frac{\left(T-t_{0}\right)^{2 q-1}}{\Gamma(q)(2 q-1)} \int_{t_{1}}^{t_{2}} M^{*} H(m) d s \\
& 0 t_{2} . \\
&
\end{aligned}
$$


Thus, $\Phi$ maps $B_{m}$ into an equicontinuous family of functions.

Step 2.2. We show that $\Phi B_{m}$ is uniformly bounded.

From (3.1), $\|x\|_{\beta}^{2} \leq m$, by $\left(H_{1}\right)-\left(H_{2}\right)$, we obtain

$$
\begin{aligned}
\|\Phi x(t)\|^{2} \leq & \left(\sum _ { k = 0 } ^ { + \infty } \left[\prod_{i=1}^{k}\left\|b_{i}\left(\tau_{i}\right)\right\|\left\|S\left(t-t_{0}\right)\right\|\|\varphi(0)\|\right.\right. \\
& +\frac{1}{\Gamma(q)} \sum_{i=1}^{k} \prod_{j=i}^{k}\left\|b_{j}\left(\tau_{j}\right)\right\| \int_{\xi_{i-1}}^{\xi_{i}}(t-s)^{q-1}\|S(t-s)\|\left\|f\left(s, x_{s}\right)\right\| d s \\
& \left.\left.+\frac{1}{\Gamma(q)} \int_{\xi_{k}}^{t}(t-s)^{q-1}\|S(t-s)\|\left\|f\left(s, x_{s}\right)\right\| d s\right] I_{\left[\xi_{k}, \xi_{k+1}\right)}(t)\right)^{2} \\
\leq & 2 M^{2} B^{2}\|\varphi(0)\|^{2}+2 M^{2} \max \left\{1, B^{2}\right\} \frac{\left(T-t_{0}\right)^{2 q-1}}{\Gamma(q)(2 q-1)} \int_{t_{0}}^{t}\left\|f\left(s, x_{s}\right)\right\|^{2} d s
\end{aligned}
$$

Thus

$$
\begin{aligned}
E\|\Phi x(t)\|^{2} & \leq 2 M^{2} B^{2} E\|\varphi(0)\|^{2}+2 M^{2} \max \left\{1, B^{2}\right\} \frac{\left(T-t_{0}\right)^{2 q-1}}{\Gamma(q)(2 q-1)} \int_{t_{0}}^{t} E\left\|f\left(s, x_{s}\right)\right\|^{2} d s \\
& \leq 2 M^{2} B^{2} E\|\varphi(0)\|^{2}+2 M^{2} \max \left\{1, B^{2}\right\} \frac{\left(T-t_{0}\right)^{2 q}}{\Gamma(q)(2 q-1)}\left\|\alpha_{m}\right\|_{L^{1}} .
\end{aligned}
$$

This yields that the set $\left\{(\Phi x)(t),\|x\|_{\beta}^{2} \leq m\right\}$ is uniformly bounded, so $\left\{\Phi B_{m}\right\}$ is uniformly bounded, by the Arzela-Ascoli theorem, $\Phi$ maps $B_{m}$ into a precompact set in $X$.

Step 2.3. We show that $\Phi B_{m}$ is compact.

Let $t_{0}<t \leq T$ be fixed, and let $\epsilon$ be a real number satisfying $\epsilon \in\left(0, t-t_{0}\right)$, for $x \in B_{m}$, we define

$$
\begin{aligned}
\left(\Phi_{\epsilon} x\right)(t)= & \sum_{k=0}^{+\infty}\left[\prod_{i=1}^{k} b_{i}\left(\tau_{i}\right) S\left(t-t_{0}\right) \varphi(0)\right. \\
& +\frac{1}{\Gamma(q)} \sum_{i=1}^{k} \prod_{j=i}^{k} b_{j}\left(\tau_{j}\right) \int_{\xi_{i-1}}^{\xi_{i}}(t-s)^{q-1} S(t-s) f\left(s, x_{s}\right) d s \\
& \left.+\frac{1}{\Gamma(q)} \int_{\xi_{k}}^{t-\epsilon}(t-s)^{q-1} S(t-s) f\left(s, x_{s}\right) d s\right] I_{\left[\xi_{k}, \xi_{k+1}\right)}(t), \quad t \in\left(t_{0}, t-\epsilon\right)
\end{aligned}
$$

Since $S(t)$ is a compact operator, the set

$$
H_{\epsilon}(t)=\left\{\left(\Phi_{\epsilon} x\right)(t): x \in B_{m}\right\}
$$

is precompact in $X$ for every $\epsilon \in\left(0, t-t_{0}\right)$. Moreover, for every $x \in B_{m}$, we have

$$
\begin{aligned}
(\Phi x)(t)-\left(\Phi_{\epsilon} x\right)(t)= & \sum_{k=0}^{+\infty}\left[\frac{1}{\Gamma(q)} \int_{\xi_{k}}^{t}(t-s)^{q-1} S(t-s) f\left(s, x_{s}\right) d s\right] I_{\left[\xi_{k}, \xi_{k+1}\right)}(t) \\
& -\sum_{k=0}^{+\infty}\left[\frac{1}{\Gamma(q)} \int_{\xi_{k}}^{t-\epsilon}(t-s)^{q-1} S(t-s) f\left(s, x_{s}\right) d s\right] I_{\left[\xi_{k}, \xi_{k+1}\right)}(t) .
\end{aligned}
$$


By using $\left(H_{1}\right)-\left(H_{2}\right)$, condition (3.1), and $\|x\|_{\beta}^{2} \leq m$, we have

$$
E\left\|(\Phi x)(t)-\left(\Phi_{\epsilon} x\right)(t)\right\|_{t}^{2} \leq M^{2} \frac{\left(T-t_{0}\right)^{2 q-1}}{\Gamma(q)(2 q-1)} \int_{t-\epsilon}^{t} M^{*} H(m) d s .
$$

Therefore, there are precompact sets arbitrarily close to the set $\left\{(\Phi x)(t): x \in B_{m}\right\}$. Hence the set $\left\{(\Phi x)(t): x \in B_{m}\right\}$ is precompact in $X$. Therefore, $\Phi$ is a completely continuous operator.

Moreover, the set $U(\Phi)=\{x \in \beta: x=\lambda \Phi x$ for some $0<\lambda<1\}$ is bounded. Consequently, by Lemma 2.1, the operator $\Phi$ has a fixed point in $\beta$. Therefore, system (1.1) has a mild solution.

\section{Exponential stability in the quadratic mean}

In this section, we will study the exponential stability of the second moment of a mild solution of system (1.1). For an $F_{t}$-adapted process, $\Phi(t):[-r, \infty) \rightarrow R$ is almost surely continuous in $t$. For the purposes of stability, we may assume that $f(t, 0) \equiv 0$ for any $t \geq t_{0}$ so that system (1.1) admits a trivial solution. Moreover, $\Phi(t)=\varphi\left(t-t_{0}\right)$ for $t \in\left[t_{0}-r, t_{0}\right]$ and $E\|\Phi\|_{t}^{2} \rightarrow 0$ as $t \rightarrow \infty$.

Definition 4.1 System (1.1) is said to be exponentially stable in the quadratic mean if there exist positive constants $C_{1}>0$ and $\lambda>0$ such that

$$
E\|x(t)\| \leq C_{1} E\|\varphi\|^{2} e^{-\lambda\left(t-t_{0}\right)}, \quad t \geq t_{0}
$$

Now we introduce the following hypotheses used in our discussion:

$\left(H_{3}\right) \mu H(\psi) \leq H(\mu \psi)$ for all $\psi \in R^{+}$, where $\mu>1$.

$\left(H_{4}\right)\|S(t)\| \leq M e^{-r\left(t-t_{0}\right)}, t \geq t_{0}$, where $M \geq 1, r>0$.

Theorem 4.1 Assume that the hypotheses of Theorem 3.1 and $\left(H_{3}\right)-\left(H_{4}\right)$ hold. If the following inequality is satisfied, then system (1.1) is exponentially stable in the quadratic mean:

$$
M_{2} \int_{t_{0}}^{T} \delta(s) d s<\int_{c_{2}}^{\infty} \frac{d s}{H(s)}
$$

where $M_{2}=2 M^{2} \max \left\{1, B^{2}\right\} \frac{\left(T-t_{0}\right)^{2 q-1}}{\Gamma(q)(2 q-1)}, c_{2}=2 M^{2} B^{2} E\left[\|\varphi\|^{2}\right]$, and $M B \geq \frac{1}{\sqrt{2}}$.

Proof Let $\Phi$ be the map defined in Theorem 3.1. Using hypotheses $\left(H_{1}\right)-\left(H_{4}\right)$, we have

$$
\begin{aligned}
& \|x(t)\|^{2} \\
& \leq \lambda^{2}\left[\sum _ { k = 0 } ^ { + \infty } \left[\left\|\prod_{i=1}^{k} b_{i}\left(\tau_{i}\right)\right\|\left\|S\left(t-t_{0}\right)\right\|\|\varphi(0)\|\right.\right. \\
& \quad+\frac{1}{\Gamma(q)} \sum_{i=1}^{k}\left\|\prod_{j=i}^{k} b_{j}\left(\tau_{j}\right)\right\| \int_{\xi_{i-1}}^{\xi_{i}}(t-s)^{q-1}\left\|S(t-s) f\left(s, x_{s}\right)\right\| d s \\
& \left.\left.+\frac{1}{\Gamma(q)} \int_{\xi_{k}}^{t}(t-s)^{q-1}\left\|S(t-s) f\left(s, x_{s}\right)\right\| d s\right] I_{\left[\xi_{k}, \xi_{k+1}\right)}(t)\right]^{2}
\end{aligned}
$$




$$
\begin{aligned}
& \leq 2 \sum_{k=0}^{+\infty}\left[\left\|\prod_{i=1}^{k} b_{i}\left(\tau_{i}\right)\right\|^{2} M^{2} e^{-2 r\left(t-t_{0}\right)}\|\varphi(0)\|^{2} I_{\left[\xi_{k}, \xi_{k+1}\right)}(t)\right] \\
& +2\left[\sum _ { k = 0 } ^ { \infty } \left[\frac{1}{\Gamma(q)} \sum_{i=1}^{k}\left\|\prod_{j=i}^{k} b_{j}\left(\tau_{j}\right)\right\| \int_{\xi_{i-1}}^{\xi_{i}}(t-s)^{q-1} M e^{-r(t-s)}\left\|f\left(s, x_{s}\right)\right\| d s\right.\right. \\
& \left.\left.+\frac{1}{\Gamma(q)} \int_{\xi_{k}}^{t}(t-s)^{q-1} M e^{-r(t-s)}\left\|f\left(s, x_{s}\right)\right\| d s\right] I_{\left[\xi_{k}, \xi_{k+1}\right)}(t)\right]^{2} \\
& \leq 2\left[\max _{k}\left\{\prod_{j=i}^{k}\left\|b_{j}\left(\tau_{j}\right)\right\|^{2}\right\}\right] M^{2} e^{-2 r\left(t-t_{0}\right)}\|\varphi(0)\|^{2} \\
& +2\left[\max _{i, k}\left\{1, \prod_{j=i}^{k}\left\|b_{j}\left(\tau_{j}\right)\right\|\right\}\right]^{2} \frac{1}{\Gamma(q)} \sum_{k=0}^{+\infty} \int_{t_{0}}^{t}(t-s)^{q-1} M e^{-r(t-s)}\left\|f\left(s, x_{s}\right)\right\| d s I_{\left[\xi_{k}, \xi_{k+1}\right)}(t)^{2} \\
& \leq 2 M^{2} B^{2} e^{-2 r\left(t-t_{0}\right)}\|\varphi(0)\|^{2}+2 M^{2} \max \left\{1, B^{2}\right\} \frac{\left(T-t_{0}\right)^{2 q-1}}{\Gamma(q)(2 q-1)} \int_{t_{0}}^{t} e^{-2 r(t-s)}\left\|f\left(s, x_{s}\right)\right\|^{2} d s, \\
& \|x\|_{t}^{2} \leq 2 M^{2} B^{2} e^{-2 r\left(t-t_{0}\right)}\|\varphi\|^{2}+2 M^{2} \max \left\{1, B^{2}\right\} \frac{\left(T-t_{0}\right)^{2 q-1}}{\Gamma(q)(2 q-1)} \int_{t_{0}}^{t} e^{-2 r(t-s)}\left\|f\left(s, x_{s}\right)\right\|^{2} d s, \\
& E\|x\|_{t}^{2} \\
& \leq 2 M^{2} B^{2} e^{-2 r\left(t-t_{0}\right)} E\left[\|\varphi\|^{2}\right] \\
& +2 M^{2} \max \left\{1, B^{2}\right\} \frac{\left(T-t_{0}\right)^{2 q-1}}{\Gamma(q)(2 q-1)} \int_{t_{0}}^{t} e^{-2 r(t-s)} \delta(s) H\left(E\left[\|x\|_{s}^{2}\right]\right) d s \\
& =2 M^{2} B^{2} e^{-2 r\left(t-t_{0}\right)} E\left[\|\varphi\|^{2}\right] \\
& +2 M^{2} \max \left\{1, B^{2}\right\} \frac{\left(T-t_{0}\right)^{2 q-1}}{\Gamma(q)(2 q-1)} e^{-2 r\left(t-t_{0}\right)} \int_{t_{0}}^{t} e^{2 r\left(s-t_{0}\right)} \delta(s) H\left(E\left[\|x\|_{s}^{2}\right]\right) d s, \\
& e^{2 r\left(t-t_{0}\right)} E\|x\|_{t}^{2} \\
& \leq 2 M^{2} B^{2} E\left[\|\varphi\|^{2}\right] \\
& +2 M^{2} \max \left\{1, B^{2}\right\} \frac{\left(T-t_{0}\right)^{2 q-1}}{\Gamma(q)(2 q-1)} \int_{t_{0}}^{t} \delta(s) H\left(e^{2 r\left(s-t_{0}\right)} E\left[\|x\|_{s}^{2}\right]\right) d s, \\
& \sup _{t_{0} \leq v \leq t} e^{2 r\left(v-t_{0}\right)} E\|x\|_{v}^{2} \\
& \leq 2 M^{2} B^{2} E\left[\|\varphi\|^{2}\right] \\
& +2 M^{2} \max \left\{1, B^{2}\right\} \frac{\left(T-t_{0}\right)^{2 q-1}}{\Gamma(q)(2 q-1)} \int_{t_{0}}^{t} \delta(s) H\left(\sup _{t_{0} \leq v \leq s} e^{2 r\left(v-t_{0}\right)} E\left[\|x\|_{v}^{2}\right]\right) d s .
\end{aligned}
$$

Let

$$
\ell_{1}(t)=\sup _{t_{0} \leq v \leq t} e^{2 r\left(v-t_{0}\right)} E\|x\|_{v}^{2}, \quad t \in\left[t_{0}, T\right]
$$

Then, for any $t \in\left[t_{0}, T\right]$, it follows that

$$
\ell_{1}(t) \leq 2 M^{2} B^{2} E\left[\|\varphi\|^{2}\right]+2 M^{2} \max \left\{1, B^{2}\right\} \frac{\left(T-t_{0}\right)^{2 q-1}}{\Gamma(q)(2 q-1)} \int_{t_{0}}^{t} \delta(s) H\left(\ell_{1}(s)\right) d s .
$$


Denoting by $u_{1}(t)$ the right-hand side of the above inequality, we obtain that

$$
\begin{aligned}
& \ell_{1}(t) \leq u_{1}(t), \quad t \in\left[t_{0}, T\right], \\
& u_{1}\left(t_{0}\right)=2 M^{2} B^{2} E\|\varphi\|^{2}=c_{2}
\end{aligned}
$$

and

$$
\begin{aligned}
u_{1}^{\prime}(t) & =2 M^{2} \max \left\{1, B^{2}\right\} \frac{\left(T-t_{0}\right)^{2 q-1}}{\Gamma(q)(2 q-1)} \delta(t) H\left(\ell_{1}(t)\right) \\
& \leq 2 M^{2} \max \left\{1, B^{2}\right\} \frac{\left(T-t_{0}\right)^{2 q-1}}{\Gamma(q)(2 q-1)} \delta(t) H\left(u_{1}(t)\right), \quad t \in\left[t_{0}, T\right] .
\end{aligned}
$$

Then

$$
\frac{u_{1}^{\prime}(t)}{H\left(u_{1}(t)\right)} \leq 2 M^{2} \max \left\{1, B^{2}\right\} \frac{\left(T-t_{0}\right)^{2 q-1}}{\Gamma(q)(2 q-1)} \delta(t), \quad t \in\left[t_{0}, T\right] .
$$

Integrating the above inequality from $t_{0}$ to $t$ and making use of the change of variable, we obtain

$$
\begin{aligned}
\int_{u_{1}\left(t_{0}\right)}^{u_{1}(t)} \frac{d s}{H(s)} & \leq 2 M^{2} \max \left\{1, B^{2}\right\} \frac{\left(T-t_{0}\right)^{2 q-1}}{\Gamma(q)(2 q-1)} \int_{t_{0}}^{t} \delta(s) d s \\
& \leq 2 M^{2} \max \left\{1, B^{2}\right\} \frac{\left(T-t_{0}\right)^{2 q-1}}{\Gamma(q)(2 q-1)} \int_{t_{0}}^{T} \delta(s) d s \\
& <\int_{c_{2}}^{\infty} \frac{d s}{H(s)}=\int_{u_{1}\left(t_{0}\right)}^{\infty} \frac{d s}{H(s)}, \quad t \in\left[t_{0}, T\right] .
\end{aligned}
$$

From the above inequality and by the mean value theorem, there is a constant $K_{1}$ such that $u_{1}(t) \leq K_{1}$, and hence $\ell_{1}(t) \leq K_{1}$. Since $\sup _{t_{0} \leq v \leq t} e^{2 r\left(v-t_{0}\right)} E\|x\|_{v}^{2}=\ell_{1}(t)$ holds for every $t \in\left[t_{0}, T\right]$, we have $\sup _{t_{0} \leq v \leq t} e^{2 r\left(v-t_{0}\right)} E\|x\|_{v}^{2} \leq K_{1}$, where $K_{1}$ depends only on $\delta$ and $\mathrm{H}$. Consequently,

$$
e^{2 r\left(t-t_{0}\right)} E\|x\|_{B}^{2}=\sup _{t_{0} \leq v \leq T} e^{2 r\left(v-t_{0}\right)} E\|x\|_{v}^{2} \leq K_{1} .
$$

In the following, we proceed as in the previous theorem. Now, we will show that $\Phi$ is a completely continuous operator using a two-step proof.

Step 1. We prove that $\Phi$ is continuous.

Let $\left\{x_{n}\right\}$ be a convergent sequence of elements of $x$ in $\beta$. Then, for each $t \in\left[t_{0}, T\right]$, we have

$$
\begin{aligned}
& E\left\|\Phi x_{n}-\Phi x\right\|_{t}^{2} \\
& \quad \leq M^{2} \max \left\{1, B^{2}\right\} \frac{\left(T-t_{0}\right)^{2 q-1}}{\Gamma(q)(2 q-1)} e^{-2 r\left(t-t_{0}\right)} \int_{t_{0}}^{t} e^{2 r\left(s-t_{0}\right)} E\left\|f\left(s, x_{n_{s}}\right)-f\left(s, x_{s}\right)\right\|^{2} d s \\
& \quad \rightarrow 0, \quad \text { as } n \rightarrow \infty .
\end{aligned}
$$

Thus $\Phi$ is continuous. 
Step 2 . We prove that $\Phi$ is a completely continuous operator.

Denote

$$
B_{m_{1}}=\left\{x \in \beta \mid\|x\|_{\beta}^{2} \leq m_{1}\right\}
$$

for some $m_{1} \geq 0$.

Step 2.1. We show that $\Phi$ maps $B_{m_{1}}$ into an equicontinuous family.

Let $x \in B_{m}$ and let $t_{1}, t_{2} \in\left[t_{0}, T\right]$. If $t_{0}<t_{1}<t_{2}<T$, then by using $\left(H_{1}\right)-\left(H_{4}\right)$ and condition (4.1) and then by following a process similar to Step 2.1 of Theorem 3.1, we get

$$
E\left\|\Phi x\left(t_{2}\right)-\Phi x\left(t_{1}\right)\right\|^{2} \rightarrow 0 \quad \text { as } t_{2} \rightarrow t_{1} .
$$

Thus, $\Phi$ maps $B_{m_{1}}$ into an equicontinuous family of functions.

Step 2.2. We show that $\Phi B_{m_{1}}$ is uniformly bounded.

From condition (4.1) and $\left(H_{1}\right)-\left(H_{4}\right)$, we obtain

$$
\begin{aligned}
\|\Phi x(t)\|^{2} \leq & 2\left[\max _{k}\left\{\prod_{j=i}^{k}\left\|b_{j}\left(\tau_{j}\right)\right\|^{2}\right\}\right] M^{2} e^{-2 r\left(t-t_{0}\right)}\|\varphi(0)\|^{2} \\
& +2\left[\max _{i, k}\left\{1, \prod_{j=i}^{k}\left\|b_{j}\left(\tau_{j}\right)\right\|\right\}\right]^{2} \\
& \times\left(\frac{1}{\Gamma(q)} \sum_{k=0}^{+\infty} \int_{t_{0}}^{t}(t-s)^{q-1} M e^{-r(t-s)}\left\|f\left(s, x_{s}\right)\right\| d s\right) I_{\left[\xi_{k}, \xi_{k+1}\right)}(t)^{2} .
\end{aligned}
$$

Thus

$$
\begin{aligned}
E\|\Phi x(t)\|^{2} \leq & 2 M^{2} B^{2} e^{-2 r\left(t-t_{0}\right)} E\|\varphi(0)\|^{2} \\
& +2 M^{2} \max \left\{1, B^{2}\right\} \frac{\left(T-t_{0}\right)^{2 q-1}}{\Gamma(q)(2 q-1)} e^{-2 r\left(t-t_{0}\right)} \\
& \times \int_{t_{0}}^{t} e^{2 r\left(s-t_{0}\right)} M^{*} H(m) d s,
\end{aligned}
$$

where $M^{*}=\sup \left\{\delta(t): t \in\left[t_{0}, T\right]\right\}$. Since $e^{-2 r\left(t-t_{0}\right)} \rightarrow 0$, the right-hand side of the above inequality tends to 0 as $t \rightarrow \infty$. That is,

$$
\|(\Phi x)\|_{\beta}^{2} \rightarrow 0 \quad \text { as } t \rightarrow \infty .
$$

This yields that the set $\left\{(\Phi x)(t),\|x\|_{B}^{2} \leq m_{1}\right\}$ is uniformly bounded, so $\left\{\Phi B_{m_{1}}\right\}$ is uniformly bounded.

Step 2.3. We show that $\Phi B_{m_{1}}$ is compact.

Let $t_{0}<t \leq T$ be fixed and let $\epsilon$ be a real number satisfying $\epsilon \in\left(0, t-t_{0}\right)$, for $x \in B_{m_{1}}$, we define

$$
\left(\Phi_{\epsilon} x\right)(t)=\sum_{k=0}^{+\infty}\left[\prod_{i=1}^{k} b_{i}\left(\tau_{i}\right) S\left(t-t_{0}\right) \varphi(0)\right.
$$




$$
\begin{aligned}
& +\frac{1}{\Gamma(q)} \sum_{i=1}^{k} \prod_{j=i}^{k} b_{j}\left(\tau_{j}\right) \int_{\xi_{i-1}}^{\xi_{i}}(t-s)^{q-1} S(t-s) f\left(s, x_{s}\right) d s \\
& \left.+\frac{1}{\Gamma(q)} \int_{\xi_{k}}^{t-\epsilon}(t-s)^{q-1} S(t-s) f\left(s, x_{s}\right) d s\right] I_{\left[\xi_{k}, \xi_{k+1}\right)}(t), \quad t \in\left(t_{0}, t-\epsilon\right) .
\end{aligned}
$$

Since $S(t)$ is a compact operator, the set

$$
H_{\epsilon}(t)=\left\{\left(\Phi_{\epsilon} x\right)(t): x \in B_{m_{1}}\right\}
$$

is precompact in $X$ for every $\epsilon \in\left(0, t-t_{0}\right)$.

Making use of hypotheses $\left(H_{1}\right)-\left(H_{4}\right)$, condition (4.1), and the fact that $\|x\|_{\beta}^{2} \leq m_{1}$, we have

$$
E\left\|(\Phi x)(t)-\left(\Phi_{\epsilon} x\right)(t)\right\|_{t}^{2} \leq M^{2} \frac{\left(T-t_{0}\right)^{2 q-1}}{\Gamma(q)(2 q-1)} e^{-2 r\left(t-t_{0}\right)} \int_{t-\epsilon}^{t} e^{2 r\left(s-t_{0}\right)} M^{*} H\left(E\|x\|_{s}^{2}\right) d s .
$$

Therefore, there are precompact sets arbitrarily close to the set $\left\{(\Phi x)(t): x \in B_{m_{1}}\right\}$. Hence the set $\left\{(\Phi x)(t): x \in B_{m_{1}}\right\}$ is precompact in $X$. Therefore, $\Phi$ is a completely continuous operator.

Moreover, the set $U(\Phi)=\{x \in \beta: x=\lambda \Phi x$ for some $0<\lambda<1\}$ is bounded. Consequently, by Lemma 2.1, the operator $\Phi$ has a fixed point in B. Therefore, system (1.1) has a mild solution with $\Phi(t)=\varphi\left(t-t_{0}\right)$ when $t \in\left[t_{0}-r, t_{0}\right]$ and $E\|\Phi\|_{t}^{2} \rightarrow 0$ as $t \rightarrow \infty$. This completes the proof.

\section{Example}

In this section, we provide an example to illustrate our main results. Consider the following random impulsive fractional differential system:

$$
\left\{\begin{array}{l}
{ }^{c} D_{t}^{q} u(t, x)=\frac{\partial^{2}}{\partial x^{2}} u(t, x)+a u(x, t-r) u(x, t), \quad t \neq \xi_{k} \\
u\left(x, \xi_{k}\right)=q(k) \tau_{k} u\left(x, \xi_{k}^{-}\right), \quad t=\xi_{k} \\
u(t, 0)=u(t, \pi)=0 \\
u(t, x)=\phi(t, x), \quad-r<t \leq 0,0 \leq x \leq \pi
\end{array}\right.
$$

Let $X=L^{2}([0, \pi])$ and $\tau_{k}$ be a random variable defined on $D_{k} \equiv\left(0, d_{k}\right)$ for $k=1,2, \ldots$, where $0<d_{k}<+\infty$. Furthermore, assume that $\tau_{k}$ follows the Erlang distribution, where $k=1,2, \ldots$ and $\tau_{i}$ and $\tau_{j}$ are independent of each other as $i \neq j$ for $i, j=1,2, \ldots ; q$ is a function of $k$; $\xi_{0}=t_{0}$ and $\xi_{k}=\xi_{k-1}+\tau_{k}$ for $k=1,2, \ldots$, here $t_{0} \in R_{\tau}$ is an arbitrary given real number.

Define $A$ an operator on $X$ by $A u=\frac{\partial^{2} u}{\partial x^{2}}$ with the domain $D(A)=\left\{u \in X \mid u\right.$ and $\frac{\partial u}{\partial x}$ are absolutely continuous, $\left.\frac{\partial^{2} u}{\partial x^{2}} \in X, u(0)=0=u(\pi)\right\}$.

It it well known that $A$ generates a strongly continuous semigroup $S(t)$ which is compact, analytic, and self-adjoint. Moreover, the operator $A$ can be expressed as

$$
A u=\sum_{n=1}^{\infty} n^{2}\left(u, u_{n}\right) u_{n}, \quad u \in D(A)
$$


where $u_{n}(\zeta)=\sqrt{\frac{2}{\pi}} \sin n \zeta, n \in N$ is the orthogonal set of eigenvectors of $A$, and for every $u \in X, S(t) u=\sum_{n=1}^{\infty} \exp \left(-n^{2} t\right)\left(u, u_{n}\right) u_{n}$, which satisfies

$$
\|S(t)\| \leq \exp \left(-\pi^{2}\left(t-t_{0}\right)\right), \quad t \geq t_{0}
$$

Hence $S(t)$ is a contraction semigroup.

Equation (5.1) can be reformulated as the following abstract equation in $X=L^{2}([0, \pi])$ :

$$
\begin{aligned}
& { }^{c} D_{t}^{q} z(t)=A z(t)+f\left(t, z_{t}\right), \quad t \neq \xi_{k}, t \geq \tau, \\
& z\left(\xi_{k}\right)=b_{k}\left(\tau_{k}\right) z\left(\xi_{k}^{-}\right), \quad k=1,2, \ldots, \\
& z_{t_{0}}=\varphi,
\end{aligned}
$$

where $z(t)=u(\cdot, t)$, i.e., $z(t)(x)=u(x, t), z_{t}(\theta)(x)=u(x, t+\theta), t \in\left[t_{0}, T\right], \theta \in[-r, 0], z(t, x)=$ $\varphi(t, x), t \in[-r, 0], x \in[0, \pi]$. The operator $A$ is defined as above. The functions $f:[\tau, T] \times$ $C \rightarrow X$ and $b_{k}: D_{k} \rightarrow X$ are given by

$$
f\left(t, z_{t}\right)=a u(x, t-r) u(x, t), \quad b_{k}\left(\tau_{k}\right)=q(k) \tau_{k},
$$

where $a>0$.

It is easy to check that system (5.1) has a mild solution if hypotheses $\left(H_{1}\right),\left(H_{2}\right)$ and the inequality

$$
2 \max \left\{1, B^{2}\right\} \frac{\left(T-t_{0}\right)^{2 q-1}}{(2 q-1) \Gamma(q)} \int_{t_{0}}^{T} \delta(s) d s<\int_{c_{1}}^{\infty} \frac{d s}{H(s)}
$$

hold, where $c_{1}=2 B^{2} E\|\varphi\|^{2}$.

Furthermore, by Theorem 4.1, if the following conditions hold

$$
2 \max \left\{1, B^{2}\right\} \frac{\left(T-t_{0}\right)^{2 q-1}}{\Gamma(q)(2 q-1)} \int_{t_{0}}^{T} \delta(s) d s<\int_{c_{2}}^{\infty} \frac{d s}{H(s)}
$$

where $c_{2}=2 B^{2} E\left[\|\varphi\|^{2}\right]$, then system (5.1) is exponentially stable in the quadratic mean.

\section{Funding}

This research was supported by the National Nature Science Foundation of China (No. 11371027), the Research Fund for the Doctoral Program (No. 2016QD110).

\section{Competing interests}

The authors declare that they have no competing interests.

\section{Authors' contributions}

SPZ carried out the main part of this article. Both authors read and approved the final manuscript.

\section{Author details}

'School of Mathematics and Physics, Anhui Jianzhu University, Hefei, P.R. China. ${ }^{2}$ School of Mathematics, Anhui University, Hefei, P.R. China.

\section{Publisher's Note}

Springer Nature remains neutral with regard to jurisdictional claims in published maps and institutional affiliations. 


\section{References}

1. Shu, X.-B., Shi, Y.: A study on the mild solution of impulsive fractional evolution equations. Appl. Math. Comput. 273, 465-476 (2016)

2. Suganya, S., Mallika Arjunan, M., Trujillo, J.J.: Existence results for an impulsive fractional integro-differential equation with state-dependent delay. Appl. Math. Comput. 266, 54-69 (2015)

3. Zhang, S., Jiang, W., Zhang, Z:: Exponential stability for a stochastic delay neural network with impulses. Adv. Differ. Equ. 2014, 250 (2014)

4. Wu, S.J., Meng, X.Z.: Boundedness of nonlinear differential systems with impulsive effect on random moments. Acta Math. Appl. Sin. 20, 147-154 (2004)

5. Anguraj, A., Vinodkumar, A.: Existence, uniqueness and stability results of random impulsive semilinear differential systems. Nonlinear Anal. Hybrid Syst. 4, 475-483 (2010)

6. Wu, S.J., Han, D.: Exponential stability of functional differential systems with impulsive effect on random moments. Comput. Math. Appl. 50, 321-328 (2005)

7. Anguraj, A., Wu, S., Vinodkumar, A.: The existence and exponential stability of semilinear functional differential equations with random impulses under non-uniqueness. Nonlinear Anal. 74, 331-342 (2011)

8. Gowrisankar, M., Mohankumar, P., Vinodkumar, A.: Stability results of random impulsive semilinear differential equations. Acta Math. Sci. 34B, 1055-1071 (2014)

9. Feckan, M., Zhou, Y., Wang, J.: On the concept and existence of solution for impulsive fractional differential equations. Commun. Nonlinear Sci. Numer. Simul. 17, 3050-3060 (2012)

10. Zhou, Y., Jiao, F.: Existence of mild solutions for fractional neutral evolution equations. Comput. Math. Appl. 59(3), 1063-1077 (2010). https://doi.org/10.1016/j.camwa.2009.06.026

11. Shu, X.-B., Lai, Y., Chen, Y.: The existence of mild solutions for impulsive fractional partial differential equations. Nonlinear Anal. 74, 2003-2011 (2011)

12. Shu, X.-B., Shi, Y.: A study on the mild solution of impulsive fractional evolution equations. Appl. Math. Comput. 273, 465-476 (2016)

13. Chadha, A., Pandey, D.N.: Existence results for an impulsive neutral stochastic fractional integro-differential equation with infinite delay. Nonlinear Anal. 128, 149-175 (2015)

14. Wang, J., Gamal Ibrahim, A., Feckan, M.: Nonlocal impulsive fractional differential inclusions with fractional sectorial operators on Banach spaces. Appl. Math. Comput. 257, 103-118 (2015)

15. Liu, S., Wu, X., Zhou, X., Jiang, W.: Asymptotical stability of Riemann-Liouville fractional nonlinear systems. Nonlinear Dyn. 86, 65-71 (2016)

16. Agarwal, R., Hristova, S., O'Regan, D.: p-moment exponential stability of Caputo fractional differential equations with noninstantaneous random impulses. J. Appl. Math. Comput. 55, 149-174 (2017)

17. Wang, J., Zhou, Y., Fečkan, M.: Nonlinear impulsive problems for fractional differential equations and Ulam stability. Computers and Mathematics with Applications 64(10), 3389-3405 (2012). https://doi.org/10.1016/j.camwa.2012.02.021

18. Liu, S., Zhou, X., Li, X., Jiang, W.: Stability of fractional nonlinear singular systems and its applications in synchronization of complex dynamical networks. Nonlinear Dyn. 84, 2377-2385 (2016)

19. Vinodkumar, A., Malar, K., Gowrisankar, M., Mohankumar, P.: Existence, uniqueness and stability of random impulsive fractional differential equations. Acta Math. Sci. Ser. B Engl. Ed. 36B, 428-442 (2016)

\section{Submit your manuscript to a SpringerOpen ${ }^{\circ}$ journal and benefit from:}

- Convenient online submission

- Rigorous peer review

- Open access: articles freely available online

- High visibility within the field

- Retaining the copyright to your article

Submit your next manuscript at $\gg$ springeropen.com 